\title{
- Rice
}

a SpringerOpen Journal

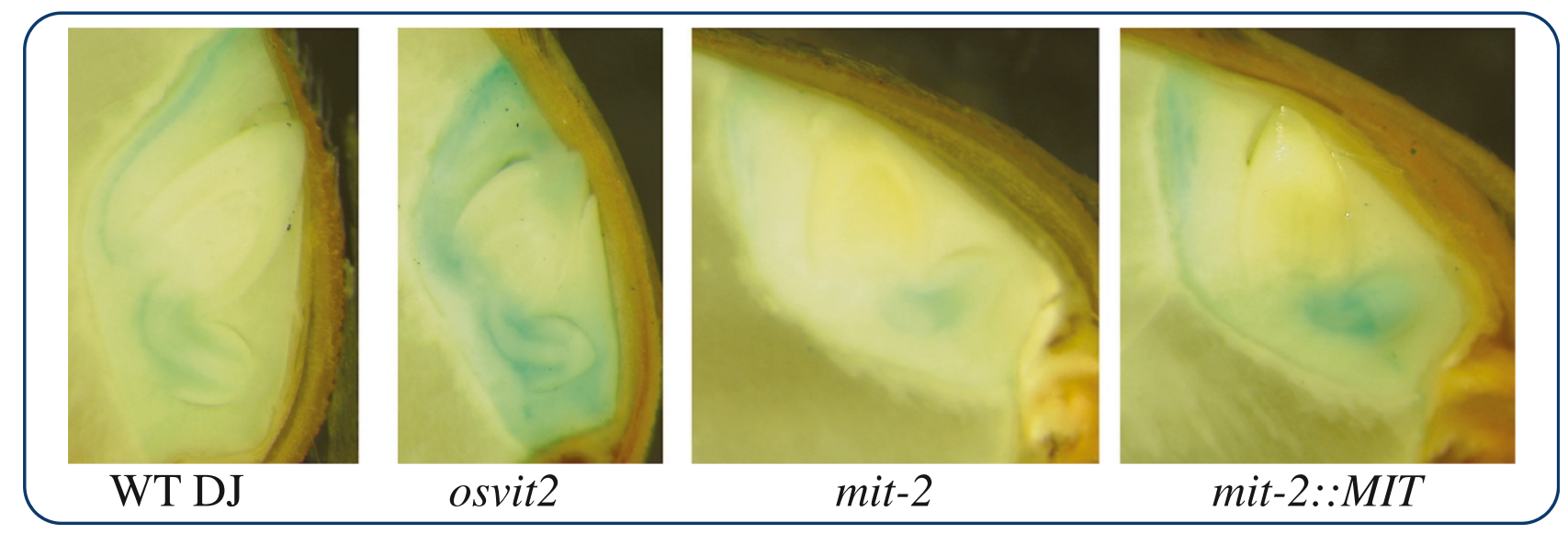

The knockdown of OsVIT2 and MIT affects iron localization in rice seed

Bashir et al. 


\title{
The knockdown of OsVIT2 and MIT affects iron localization in rice seed
}

\author{
Khurram Bashir ${ }^{1,4}$, Ryuichi Takahashi', Shamim Akhtar', Yasuhiro Ishimaru², Hiromi Nakanishi' and Naoko K Nishizawa ${ }^{1,3^{*}}$
}

\begin{abstract}
Background: The mechanism of iron (Fe) uptake in plants has been extensively characterized, but little is known about how Fe transport to different subcellular compartments affects Fe localization in rice seed. Here, we discuss the characterization of a rice vacuolar Fe transporter 2 (OsVIT2) T-DNA insertion line (osvit2) and report that the knockdown of OSVIT2 and mitochondrial Fe transporter (MIT) expression affects seed Fe localization.

Findings: osvit2 plants accumulated less Fe in their shoots when grown under normal or excess Fe conditions, while the accumulation of Fe was comparable to that in wild-type (WT) plants under Fe-deficient conditions. The accumulation of zinc, copper, and manganese also changed significantly in the shoots of osvit2 plants. The growth of osvit2 plants was also slow compared to that of WT plants. The concentration of Fe increased in osvit2 polished seeds. Previously, we reported that the expression of OsVIT2 was higher in MIT knockdown (mit-2) plants, and in this study, the accumulation of Fe in mit-2 seeds decreased significantly.
\end{abstract}

Conclusions: These results suggest that vacuolar Fe trafficking is important for plant Fe homeostasis and distribution, especially in plants grown in the presence of excess Fe. Moreover, changes in the expression of OsVIT2 and MIT affect the concentration and localization of metals in brown rice as well as in polished rice seeds.

Keywords: Iron; Manganese; Mitochondrial iron transporter; Oryza sativa; Vacuolar iron transporter; Zinc

\section{Findings}

Iron $(\mathrm{Fe})$ is an essential micronutrient for all higher organisms. Plants require Fe for several cellular processes, including respiration, chlorophyll biosynthesis, and photosynthetic electron transport (Marschner 1995). The molecular mechanism of Fe transport in rice has been well documented (Bashir et al. 2006; Bashir and Nishizawa 2006; Bashir et al. 2010; Bashir et al. 2011b; Bashir et al. 2013a; Ishimaru et al. 2012; Kobayashi and Nishizawa 2012). Once inside a plant, Fe enters root cells and is transported to the shoot and seeds. Fe performs vital roles in subcellular organelles such as chloroplasts and mitochondria, and defects in mitochondrial Fe homeostasis significantly affect plant growth (Bashir et al. 2011a; Bashir et al. 2011c; Ishimaru et al. 2009). As excess Fe in the cytoplasm may be toxic, it is either stored as ferritin

\footnotetext{
* Correspondence: annaoko@mail.ecc.u-tokyo.ac.jp

${ }^{1}$ Graduate School of Agricultural and Life Sciences, The University of Tokyo,

1-1-1 Yayoi, Bunkyo-ku, Tokyo 113-8657, Japan

${ }^{3}$ Research Institute for Bioresources and Biotechnology, Ishikawa Prefectural

University, 1-308 Suematsu, Nonoichi-shi, Ishikawa 921-8836, Japan

Full list of author information is available at the end of the article
}

in chloroplasts or is diverted to the vacuole. Knockout mutants for the rice vacuolar metal transporters OsVIT1 and OsVIT2 were recently reported to accumulate increased amounts of $\mathrm{Fe}$ in their seeds (Zhang et al. 2012). This accumulation was mainly observed in the embryo (Zhang et al. 2012), which is removed during milling. In this short report, we describe the characterization of a mutant in which the expression of OsVIT2 was knocked down and we show that changes in the expression of OsVIT2 and mitochondrial iron transporter $(M I T)$ affect seed Fe localization in brown rice as well as in polished rice seeds.

We characterized a T-DNA line (An et al. 2003a; Jeong et al. 2006) in which the T-DNA was integrated $\sim 500 \mathrm{bp}$ upstream of the start codon of OsVIT2 (Os09g0396900), as shown in Figure 1a. (For details see Additional file 1 "Methods"). Genomic polymerase chain reaction (PCR) using primers specific for the T-DNA integration site confirmed the homozygous status of the plants (Figure 1b), while primers specific for exon 3 were used to check the quality of the DNA (Figure 1c). Quantitative PCR analysis confirmed that the expression of OsVIT2 was significantly 


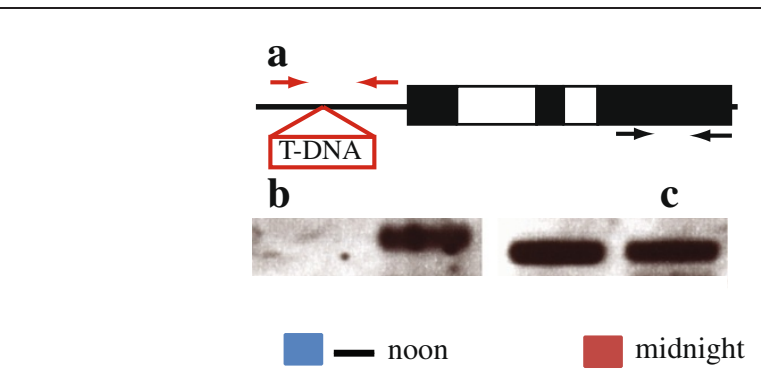

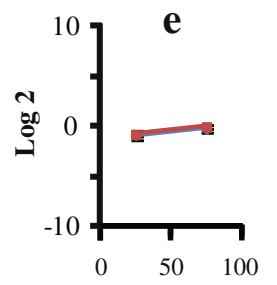

DAT
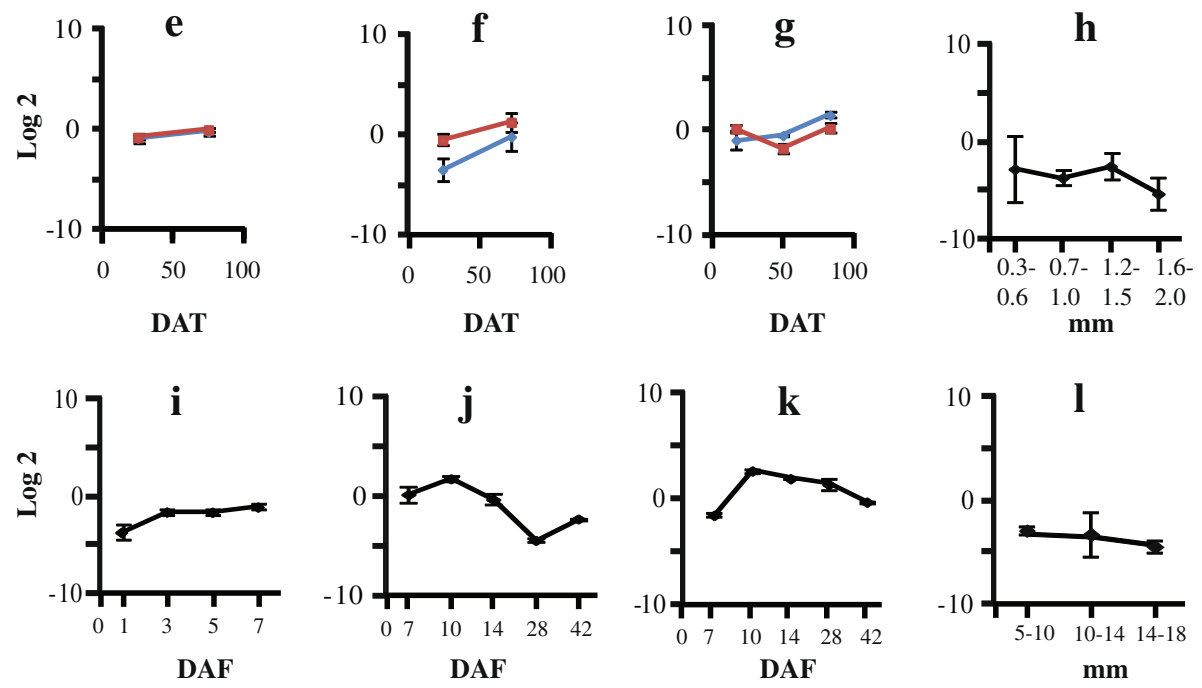

Figure 1 Characterization of the OsVIT2 knockdown mutant (Osvit2) and expression of OsVIT2 during different developmental stages of rice. a: Gene structure and positions of the primers specific for the T-DNA insertion. $\mathbf{b}$ : Bands corresponding to the primers are shown in red. c: Bands corresponding to the primers are shown black. d: Quantitative reverse-transcription PCR analysis showing the expression of Osvit2 in WT and knockdown plants. A vertical bar followed by an asterisk indicates a significant difference from WT according to the Tukey-Kramer test ( $p<0.05 ; W T, n=4 ;$ osvit2, $n=8)$. e: Roots at 27 and 76 DAT. f: Stems at 27 and 76 DAT. g: Leaf blades at 27, 76, and 125 DAT. $\mathbf{h}$ : The anther size was 0.3-0.6, 0.7-1.0, 1.2-1.5, and 1.6-2.0 mm. i: Embryos 1, 3, 5, and 7 days after flowering. j: Ovaries 7, 10, 14, 28, and 42 days after flowering. k: Endosperm, 7, 10, 14, 28, and 42 days after flowering. I: The pistil size was 5-10, 10-14, and 14-18 mm ( $\mathrm{n}=3$ ). Error bars represent the SD.

downregulated in the osvit2 line compared to wild-type (WT) plants (Figure 1d). Note that similar to indica rice, the sequence of OsVIT2 is not complete in RAP-DB (http://rapdb.dna.affrc.go.jp/) and the sequence of OsVIT2 showed $100 \%$ similarity to that reported for indica rice (Zhang et al. 2012). Data related to the expression of OsVIT2 were generated through rice global gene expression profile data sets maintained at http://ricexpro.dna. affrc.go.jp (Sato et al. 2011a; Sato et al. 2011b). OsVIT2 expression is upregulated in the presence of excess $\mathrm{Fe}$ (Bashir et al. 2011c), and the expression of OsVIT2 could be observed through all developmental stages (Figure $1 \mathrm{e}-\mathrm{l})$. In roots, the expression of OsVIT2 increased slightly from 27 days after transplantation (DAT) to 76 DAT and was not regulated diurnally (Figure 1e). A similar trend was observed in the stems (Figure 1f). In leaves, the expression of OsVIT2 increased slightly at noon from 27 to 76 DAT and at 125 DAT, while at midnight, it decreased slightly from 27 to 76 DAT and then increased at 127 DAT (Figure 1g). OsVIT2 expression did not change significantly during anther development (Figure 1h). In the embryo, OsVIT2 expression increased slightly from 1 to 3,5 , and 7 days after fertilization (DAF; Figure 1i). In ovary, OsVIT2 expression first increased from 7 to 10 DAF, then decreased from 10 to 14 and 28 DAF, and then increased again at 42 DAF (Figure 1j). In endosperm, the expression of OsVIT2 first increased from 7 to 10 DAF and then decreased from 10, 14, 28, and 42 DAF (Figure 1k). In addition, OsVIT2 expression remained largely unchanged during pistil development (Figure 11). These results clearly support the earlier results of Zhang et al. (2012) showing that OsVIT2 plays a critical role in transporting Fe and zinc ( $\mathrm{Zn}$ ) from leaves to seeds.

We grew WT and osvit2 plants under different Fe concentrations. Under Fe-deficient conditions, no difference was observed in shoot length, root length, or chlorophyll content (SPAD value; Figure 2a, d, and g), while at 100 and $500 \mu \mathrm{M}$ Fe-EDTA, shoot growth was significantly retarded compared to WT plants (Figure $2 \mathrm{~b}$ and c), whereas the root length and SPAD value remained 

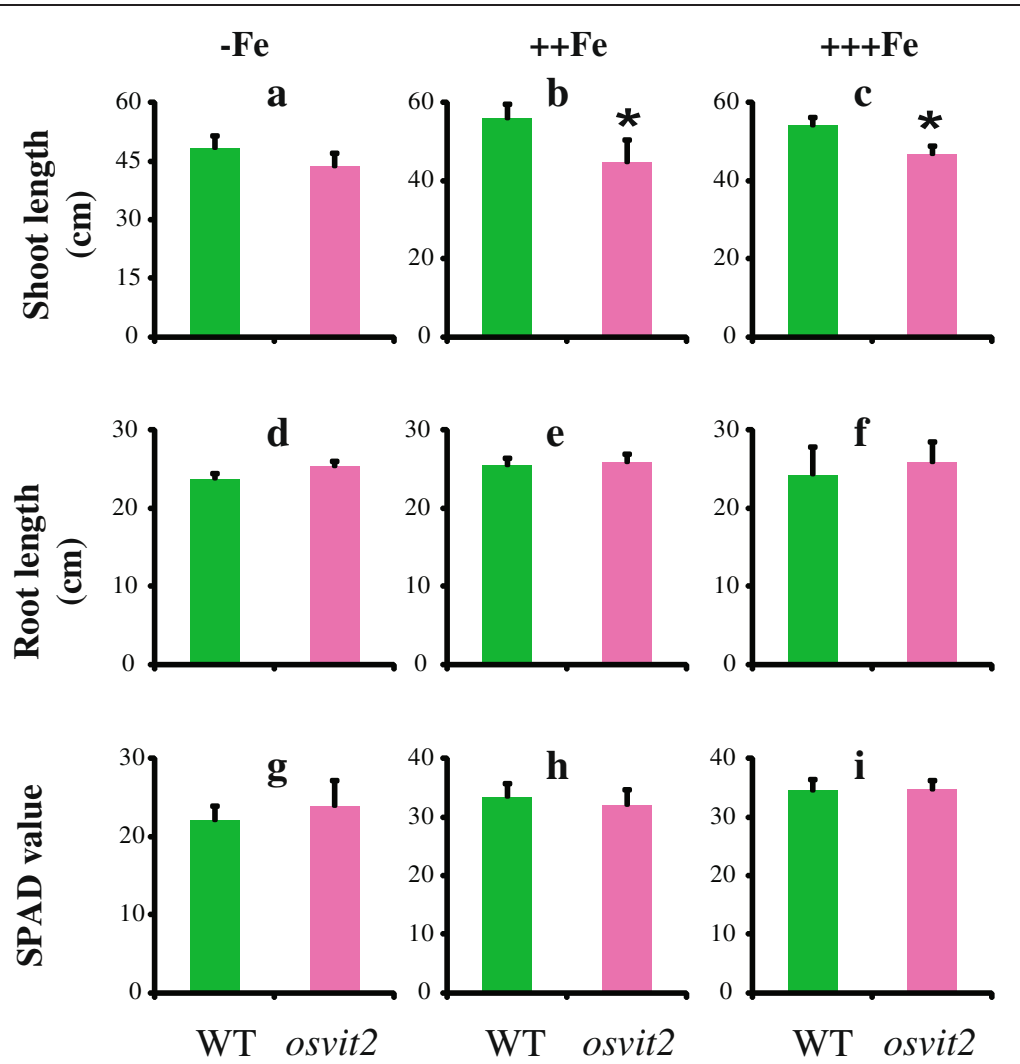

Figure 2 Morphological characteristics of osvit2 plants grown at different Fe concentrations. a-c: Shoot length. d-f: Root length. $\mathbf{g}-\mathrm{i}$ :

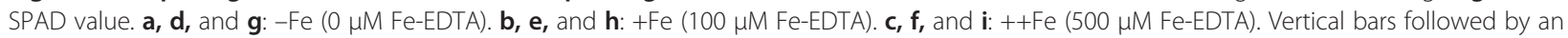
asterisk indicate a significant difference from the WT according to the Tukey-Kramer test $(p<0.05 ; n=4)$.

unchanged (Figure 2e, f, h, and i). We also measured the concentrations of $\mathrm{Fe}, \mathrm{Zn}$, manganese $(\mathrm{Mn})$, and copper $(\mathrm{Cu})$ in the roots and shoots of WT and osvit2 plants. No difference was observed between WT and osvit2 shoots for all of these metals when the plants were grown in the absence of Fe (Figure 3a, g, m, and s). When plants were grown in the presence of $100 \mu \mathrm{M}$ Fe-EDTA, the concentrations of Fe, $\mathrm{Zn}$, and Mn decreased significantly in osvit2 compared to WT plants. The concentration of $\mathrm{Cu}$ also decreased. When plants were grown under $\mathrm{Fe}$ excess conditions, the concentrations of $\mathrm{Fe}, \mathrm{Mn}$, and $\mathrm{Cu}$ decreased significantly in the shoots of osvit2 plants. The roots of osvit2 plants accumulated more $\mathrm{Fe}$ and $\mathrm{Zn}$ when grown under Fe-deficient conditions (Figure $3 \mathrm{~d}$ and $\mathrm{j}$ ), while the metal concentration in osvit2 roots was comparable to that in WT plants following growth with 100 or $500 \mu \mathrm{M}$ Fe-EDTA (Figure 3e, f, k, l, $\mathrm{p}-\mathrm{r}$, and $\mathrm{v}-\mathrm{x}$ ). Pearl staining analysis showed that osvit2 plants accumulated more Fe in their embryos compared to WT plants (Figure 4a and b). Mutants for AtVIT1 (Kim et al. 2006) and OsVIT1 and OsVIT2 (Zhang et al. 2012) have been reported to have disturbed Fe accumulation in seeds.
We previously reported that in mit-2 plants, the expression of OsVIT2 was upregulated (Bashir et al. 2011c), so we assessed whether the concentration of $\mathrm{Fe}$ and other metals also changed in mit-2 seeds. mit-2 seeds accumulated less Fe in the embryo compared to WT plants, and in mit-2 lines complemented with MIT, the localization of Fe was comparable to that in WT plants. We further measured the concentration of different metals in mit-2 and osvit2 seeds. The concentrations of $\mathrm{Fe}, \mathrm{Zn}$, and $\mathrm{Cu}$ increased in osvit2 seeds, while that of $\mathrm{Mn}$ decreased (Figure 5). As we previously reported that the expression of OsVIT2 was upregulated in mit-2 plants (Bashir et al. 2011c), we analyzed mit-2 seeds for changes in metal accumulation. The concentration of Fe was higher in leaves harvested from mit-2 plants. A knockout line of osvit2 was previously shown to accumulate an increased amount of $\mathrm{Fe}$ in its seeds (Zhang et al. 2012); however, in that report, the authors did not analyze the metal concentration in polished rice. As the embryo is removed during milling, leaving the endosperm as the only edible part, we measured the concentration of $\mathrm{Fe}$ and other metals in polished rice seeds (rice endosperm). In polished osvit2 seeds, the concentrations of $\mathrm{Fe}, \mathrm{Zn}$, and 

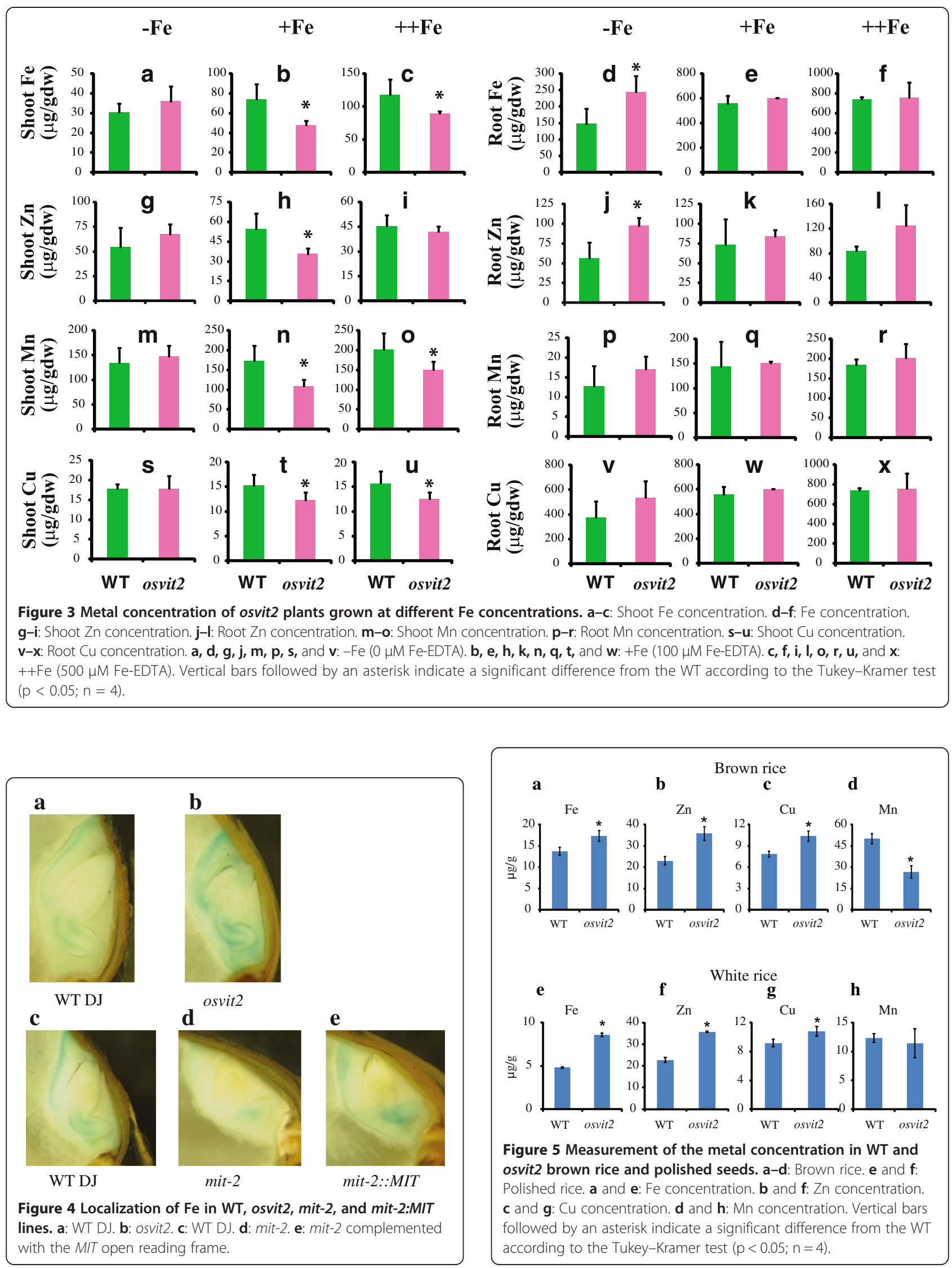
$\mathrm{Cu}$ were significantly elevated compared to WT seeds (Figure 5e-g). Rice is an important agronomical crop and is used as a staple food by approximately half of the world's population. Rice is poor in nutrients such as Fe, and people who depend on rice as a staple food often suffer from Fe deficiency (Bashir et al. 2013b; Bashir et al. 2010). Fe and $\mathrm{Zn}$ deficiencies cause 0.8 million deaths annually, while the number of people suffering from these deficiencies is up to 2 billion (World Health Organization 2003). Thus, breeding rice plants that are capable of accumulating more $\mathrm{Fe}$ and $\mathrm{Zn}$ in the endosperm is important (Bashir et al. 2012; Bashir et al. 2013b). These results indicate that the knockout/ knockdown of osvit2 may be utilized for biofortification programs. In mit-2 seeds, Fe accumulation was significantly lower compared to WT plants (Figure 6a), while the concentration of other metals did not change significantly (Figure 6a-d). Polished mit-2 seeds also accumulated significantly less Fe, while they accumulated more Zn compared to WT seeds (Figure 6e and f); no difference was observed in other metals. OsVIT2 overexpression lines accumulated less Fe in their seeds, and in mit-2,
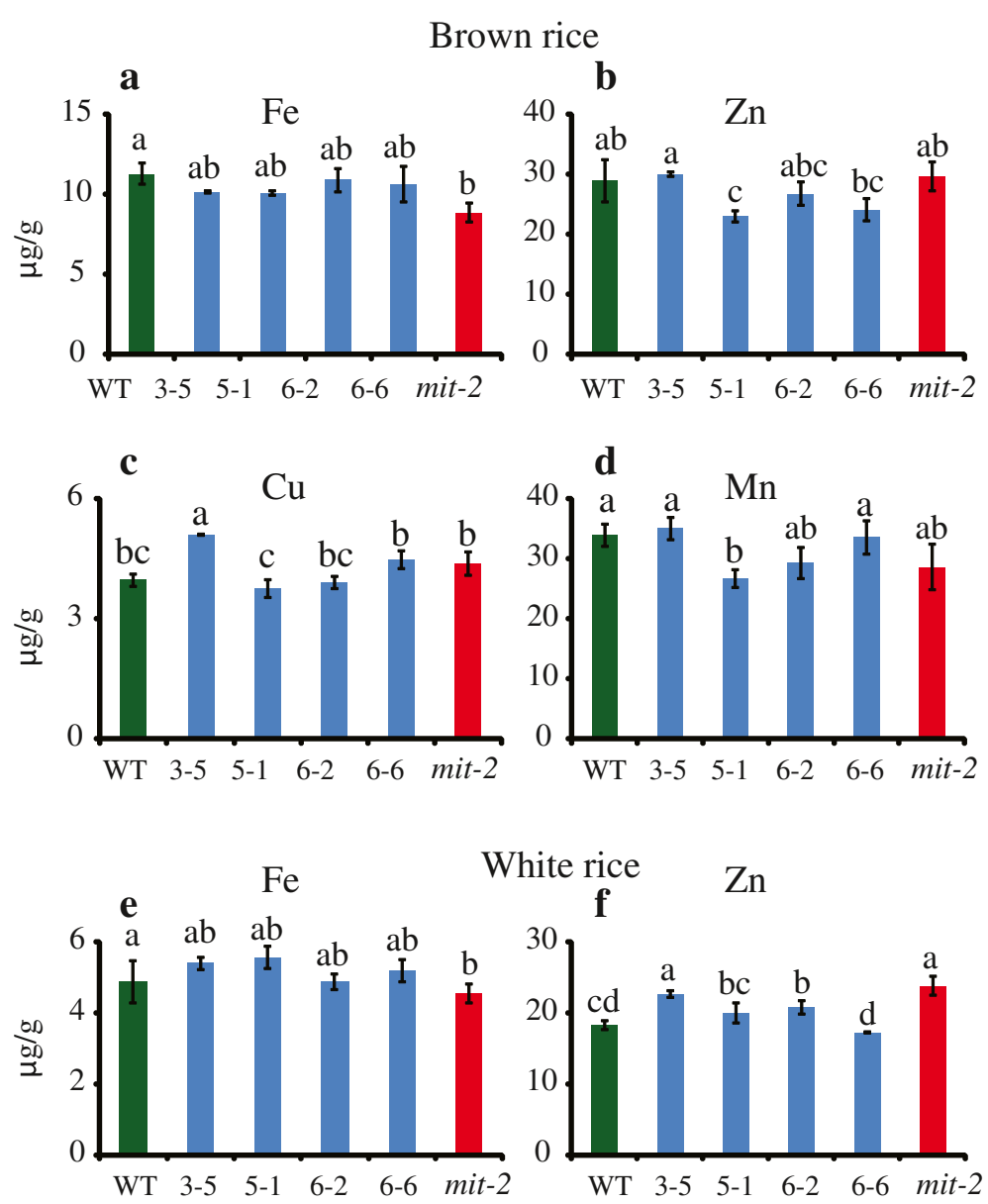

White rice $\mathrm{Zn}$
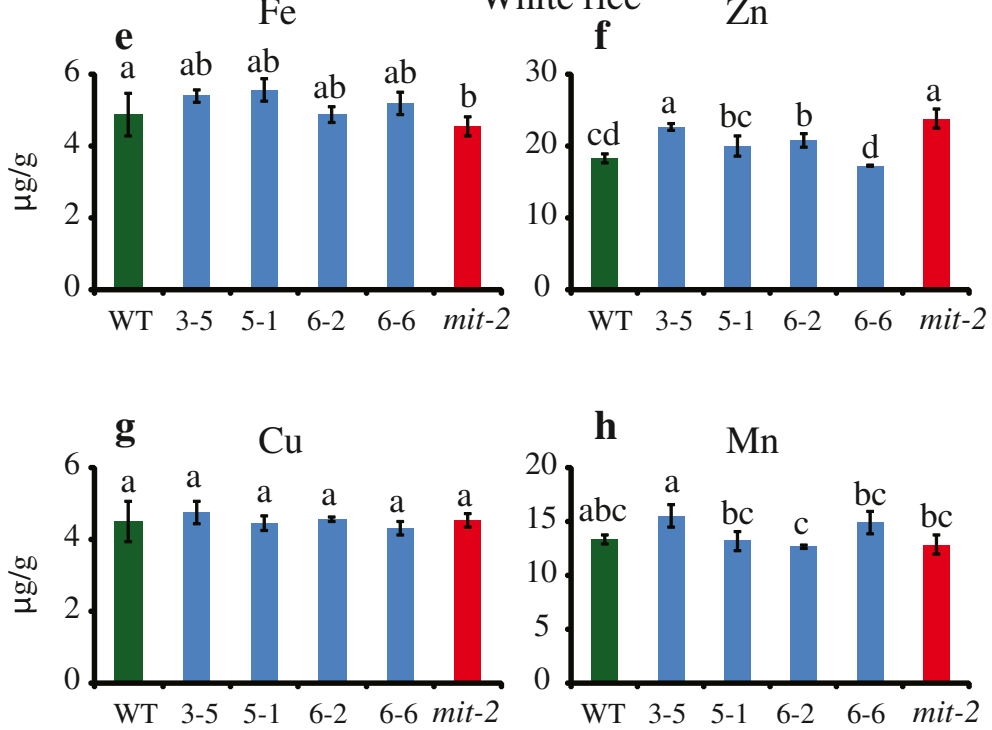

Figure 6 Measurement of the metal concentration in WT and mit-2 brown rice and polished seeds. a-d: Brown rice. e and f: Polished rice. $\mathbf{a}$ and $\mathbf{e}$ : Fe concentration. $\mathbf{b}$ and $\mathbf{f}$ : Zn concentration. $\mathbf{c}$ and $\mathbf{g}$ : Cu concentration. $\mathbf{d}$ and $\mathbf{h}$ : Mn concentration. Vertical bars followed by different letters are significantly different from each other according to the Tukey-Kramer test $(p<0.05 ; n=4)$. 
the reduction in $\mathrm{Fe}$ accumulation may be caused by increased OsVIT2 expression. Signaling between different subcellular organelles (Vigani et al. 2013) may be responsible for changes in metal localization in rice seeds. These results suggest that subcellular Fe transporters affect seed Fe localization; thus, it may be possible to regulate the expression of these transporters to biofortify rice with $\mathrm{Fe}$ and $\mathrm{Zn}$ without causing any adverse effects on plant growth and development.

\section{Additional file}

\section{Additional file 1: Methods.}

\section{Competing interests}

The authors declare no potential competing interests.

\section{Authors' contributions}

$\mathrm{KB}$ and NKN conceived and designed the experiments. SA screened the osvit2 line. KB performed the experiments. KB, RT, YI, HN, and NKN discussed the data and wrote the paper. All authors read and approved the final manuscript.

\section{Acknowledgments}

This study was supported by a grant from the Ministry of Agriculture, Forestry and Fisheries of Japan (Green Technology Project IP-5003).

\section{Accession codes}

In our previous report (Bashir et al. 2011c), OsVIT2 was referred to as OsVIT1, while Zhang et al. (2012) used the name OsVIT2 for the same gene. To avoid confusion, in this study we changed the name of OsVIT1 to OsVIT2.

\section{Author details}

${ }^{1}$ Graduate School of Agricultural and Life Sciences, The University of Tokyo, 1-1-1 Yayoi, Bunkyo-ku, Tokyo 113-8657, Japan. ${ }^{2}$ Faculty of Science, Graduate School of Science, Tohoku University, 6-3 Aramakiaza-aoba, Aoba-ku, Sendai, Miyagi 980-8578, Japan. ${ }^{3}$ Research Institute for Bioresources and Biotechnology, Ishikawa Prefectural University, 1-308 Suematsu, Nonoichi-shi, Ishikawa 921-8836, Japan. ${ }^{4}$ Present Address: Plant Genomic Network Research Team, Center for Sustainable Resource Sciences, RIKEN Yokohama Campus, 1-7-22, Suehiro-cho, Tsurumi-ku, Yokohama, Kanagawa 230-0045, Japan.

Received: 14 May 2013 Accepted: 12 September 2013 Published: 20 November 2013

\section{References}

An S, Park S, Jeong D-H, Lee D-Y, Kang H-G, Yu J-H, Hur J, Kim S-R, Kim Y-H, Lee M, Han S, Kim S-J, Yang J, Kim E, Wi SJ, Chung HS, Hong J-P, Choe V, Lee H-K, Choi J-H, Nam J, Kim S-R, Park P-B, Park KY, Kim WT, Choe S, Lee C-B, An G (2003a) Generation and analysis of end aequence satabase for T-DNA tagging lines in rice. Plant Physiol 133(4):2040-2047, doi:10.1104/pp. 103.030478

Bashir K, Nishizawa NK (2006) Deoxymugineic acid synthase: a gene important for fe-acquisition and homeostasis. Plant Signal Behav 1(6):290-292

Bashir K, Inoue H, Nagasaka S, Takahashi M, Nakanishi H, Mori S, Nishizawa NK (2006) Cloning and characterization of deoxymugineic acid synthase genes from graminaceous plants. J Biol Chem 281(43):32395-32402, doi:10.1074/jbc. M604133200

Bashir K, Ishimaru Y, Nishizawa NK (2010) Iron uptake and loading into rice grains. Rice 3(2):122-130, doi:10.1007/s12284-010-9042-y

Bashir K, Ishimaru Y, Nishizawa NK (2011a) Identification and characterization of the major mitochondrial Fe transporter in rice. Plant Signal Behav 6(10):1591-1593

Bashir K, Ishimaru Y, Shimo H, Kakei Y, Senoura T, Takahashi R, Sato Y, Sato Y, Uozumi N, Nakanishi H, Nishizawa NK (2011b) Rice phenolics efflux transporter 2 (PEZ2) plays an important role in solubilizing apoplasmic iron. Soil Sci Plant Nutr 57(6):803-812, doi:10.1080/00380768.2011.637305
Bashir K, Ishimaru Y, Shimo H, Nagasaka S, Fujimoto M, Takanashi H, Tsutsumi N, An G, Nakanishi H, Nishizawa NK (2011c) The rice mitochondrial iron transporter is essential for plant growth. Nat Commun 2:322, doi:10.1038/ncomms1326

Bashir K, Ishimaru Y, Nishizawa NK (2012) Molecular mechanisms of zinc uptake and translocation in rice. Plant Soil 361:189-201, doi:10.1007/s11104-012-1240-5

Bashir K, Nozoye T, Ishimaru Y, Nakanishi H, Nishizawa NK (2013a) Exploiting new tools for iron bio-fortification of rice: biotechnology advances. 31(8):16241633, doi:http://dx.doi.org/10.1016/j.biotechadv.2013.08.012

Bashir K, Takahashi R, Nakanishi H, Nishizawa NK (2013b) The road to micronutrient biofortification of rice: progress and prospects. Front Plant Sci 4(15), doi:10.3389/fpls.2013.00015

Ishimaru Y, Bashir K, Fujimoto M, An G, Itai RN, Tsutsumi N, Nakanishi H, Nishizawa NK (2009) Rice-specific mitochondrial iron-regulated gene (MIR) plays an important role in iron homeostasis. Mol Plant 2(5):1059-1066, doi:10.1093/mp/ssp051

Ishimaru Y, Takahashi R, Bashir K, Shimo H, Senoura T, Sugimoto K, Ono K, Yano M, Ishikawa S, Arao T, Nakanishi H, Nishizawa NK (2012) Characterizing the role of rice NRAMP5 in manganese, iron and cadmium transport. Sci Rep 2 doi:10.1038/srep00286

Jeong D-H, An S, Park S, Kang H-G, Park G-G, Kim S-R, Sim J, Kim Y-O, Kim M-K, Kim S-R, Kim J, Shin M, Jung M, An G (2006) Generation of a flanking sequence-tag database for activation-tagging lines in japonica rice. Plant J 45(1):123-132, doi:10.1111/j.1365-313X.2005.02610.x

Kim SA, Punshon T, Lanzirotti A, Li L, Alonso JM, Ecker JR, Kaplan J, Guerinot ML (2006) Localization of iron in Arabidopsis seed requires the vacuolar membrane transporter VIT1. Science 314(5803):1295-1298, doi:10.1126/science.1132563

Kobayashi T, Nishizawa NK (2012) Iron uptake, translocation, and regulation in higher plants. Annu Rev Plant Biol 63(1):131-152, doi:10.1146/annurev-arplant-042811105522

Marschner H (1995) Mineral nutrition of higher plants, 2nd edn. Academic Press, London

Sato Y, Antonio B, Namiki N, Motoyama R, Sugimoto K, Takehisa H, Minami H, Kamatsuki K, Kusaba M, Hirochika H, Nagamura Y (2011a) Field transcriptome revealed critical developmental and physiological transitions involved in the expression of growth potential in japonica rice. BMC Plant Biol 11(1):10

Sato Y, Antonio BA, Namiki N, Takehisa H, Minami H, Kamatsuki K, Sugimoto K, Shimizu Y, Hirochika H, Nagamura Y (2011b) RiceXPro: a platform for monitoring gene expression in japonica rice grown under natural field conditions. Nucleic Acids Res 39(suppl 1):D1141-D1148, doi:10.1093/nar/gkq1085

Vigani G, Zocchi G, Bashir K, Philippar K, Briat J-F (2013) Signals from chloroplasts and mitochondria for iron homeostasis regulation. Trends Plant Sci 18 (6):305-311, doi:http://dx.doi.org/10.1016/j.tplants.2013.01.006

World Health Organization (2003) Summary and conclusion of the sixty-first meeting of the joint FAO/WHO expert committee on food additives. World Health Organization, http://www.who.int/foodsafety/chem/jecfa/summaries/ en/summary_61.pdf

Zhang Y, Xu Y-H, Yi H-Y, Gong J-M (2012) Vacuolar membrane transporters OsVIT1 and OsVIT2 modulate iron translocation between flag leaves and seeds in rice. Plant J 72:400-410, doi:10.1111/j.1365-313X.2012.05088.X

doi:10.1186/1939-8433-6-31

Cite this article as: Bashir et al:: The knockdown of OsVIT2 and MIT affects iron localization in rice seed. Rice 2013 6:31.

\section{Submit your manuscript to a SpringerOpen ${ }^{\circ}$ journal and benefit from:}

- Convenient online submission

Rigorous peer review

- Immediate publication on acceptance

- Open access: articles freely available online

- High visibility within the field

- Retaining the copyright to your article

Submit your next manuscript at $>$ springeropen.com 